Universidad de Panamá
Instituto de Estudios Nacionales
ISNN 1810-5491

Cuadernos Nacionales

$\mathrm{N} *$ 26, 54-71, Enero-Junio, 2020

\title{
Derecho al juego, educación y cuidado infantil en familias indígenas migrantes del municipio de Ascensión, estado de Chihuahua ${ }^{1}$
}

\author{
Dr. Pavel Roel Gutiérrez Sandoval ${ }^{2}$ \\ Dra. Evangelina Cervantes Holguín ${ }^{3}$ \\ Dr. Carlos Sandino Gutiérrez Sandoval ${ }^{4}$ \\ Universidad Autónoma de Ciudad Juárez
}

\section{Resumen}

Se emplea el método fenomenológico, la (micro)etnografía y el método biográfico bajo el paradigma comprensivo para dar sustento metodológico al análisis cualitativo del derecho al juego, el acceso a la educación y las condiciones del cuidado infantil de las niñas, niños y adolescentes (NNA) indígenas migrantes en la sierra limítrofe entre el estado de Chihuahua y el estado de Sonora, Norte de México. Así, se triangulan los resultados de la entrevista personal, las crónicas de experiencias laborales y la observación de las interacciones entre las NNA como fuente metodológica para aportar conocimiento sobre la irrupción que provocan la explotación laboral infantil, la falta de estimulación temprana o la exclusión de los procesos educativos sobre las tareas del desarrollo y formación integral de las NNA. Se pone mayor énfasis en la ausencia de una política migratoria integral desde el paradigma de justicia y no solo desde el discurso de los derechos humanos. Se coincide en la necesidad de una ley de migración indígena que garantice que las familias indígenas migrantes renuncien al nomadismo, evite la victimización bajo el estereotipo de grupo vulnerable y garantice la protección, la difusión de las tradiciones culturales indígenas y la inclusión social para que las NNA puedan tener una vida libre de violencia y una participación ciudadana completa

1 Recibido 9/16/2019 - Aprobado 11/15/2019.

2 Doctor en Educación. Profesor-investigador con reconocimiento por el Consejo Nacional de Ciencia y Tecnología (Conacyt, SNI nivel I) adscrito al Programa de Maestría en Estudios Interdisciplinarios de Género y Programa de Licenciatura en Educación de la Universidad Autónoma de Ciudad Juárez (UACJ), pavel.gutierrez@uacj.mx

3 Doctora en Educación. Profesora-Investigadora adscrita al Programa de Maestría en Investigación Educativa Aplicada de la UACJ. Coordinadora del Centro Chihuahuense de Estudios de Posgrado (CCHEP), Unidad Juárez, evangelina.cervantes@uacj.mx

4 Doctor en Educación. Colaborador en proyectos de investigación de miembros del Cuerpo Académico 54: Estudios de Educación y Ciencias Sociales de la Universidad Autónoma de Ciudad Juárez (UACJ), carlos.gutierrez@uacj.mx 
(informada, consciente, acciones iniciadas por ellas/os y con decisiones tomadas con el apoyo de las autoridades). Es importante reconocer la importancia de que las políticas públicas para las NNA migrantes indígenas necesitan enfocarse la atención psicoconductual, el desarrollo emocional, la re-territorialización, los derechos humanos y, en específico, el derecho al esparcimiento y el juego.

Palabras clave: NNA indígenas migrantes; Derecho al juego; Trabajo infantil; Dificultades de aprendizaje; Exclusión social

\begin{abstract}
The phenomenological, the ethnography and the biographical method are used under the comprehensive paradigm to give methodological support to the qualitative analysis of the right to play, access to education and the conditions of child care of indigenous migrant children and adolescents (NNAs) in the bordering between the state of Chihuahua and the state of Sonora, Northern Mexico. Thus, the results of the personal interview, the chronicles of work experiences and the participant observation of the interactions between the children as a methodological source are triangulated to provide knowledge about the irruption caused by child labor exploitation, lack of early stimulation or exclusion of the educational processes on the tasks of the development and integral formation of the children. Greater emphasis is placed on the absence of a comprehensive immigration policy from the justice paradigm and not only from the discourse of human rights. The need for an indigenous migration law is agreed to ensure that migrant indigenous families renounce nomadism, avoid victimization under the stereotype of a vulnerable group and guarantee protection, dissemination of indigenous cultural traditions and social inclusion so that Children can have a life free of violence and full citizen participation (informed, aware, actions initiated by them and with decisions taken with the support of the authorities). It is important to recognize the importance of public policies for indigenous migrant children being directed to psychobehavioral attention, emotional development, re-territorialization, human rights and, specifically, the right to recreation and play.
\end{abstract}

Keywords: Indigenous migrant children; Right to play; Child labor; Learning difficulties; Social exclusion

\title{
Introducción
}

Este artículo presenta solo una parte de los resultados de un proyecto de investigación más amplio que tiene como propósito de visibilizar las injusticias que marcan la vida de las niñas, niños y adolescentes (NNA) indígenas migrantes y conocer desde sus propias voces las situaciones respecto al derecho al juego, el acceso a la educación y los cuidados. Se considera un desplazamiento nómada, es decir, en tránsito dentro de una migración cíclica relacionada con el trabajo infantil en huertas de cultivo que, entre otras entidades federativas, atraviesa a la región limítrofe entre el estado de Chihuahua y el estado de Sonora, incluyendo las colonias de reciente creación Ejidos o Secciones localizadas en los municipios de: Bacerac, Bavispe y Agua Prieta, Son. y Casas Grandes, Janos y Ascensión, Chih. 
Velasco y Rentería (2019) sostienen que la complejidad de la migración no oculta la diferenciación étnica colonial, la discriminación racial y los prejuicios contra los pueblos indígenas. En 1982, la presencia de inmigrantes indígenas en Baja California llevó a la transferencia de maestros del sistema educativo indígena de Oaxaca que se unieron al magisterio local para expandir el sistema educativo del estado. Por lo tanto, entre 1982 y 1999, el número de estudiantes aumentó once veces su tamaño, de 692 a 7,754, mientras que el número de maestros aumentó a nueve veces, de 33 a 294 (Lestage y Pérez, 2000, citado en Velasco y Rentería, p. 7). Primero, se hace un llamado a reconsiderar la creencia de que la educación indígena solo está dirigida para personas indígenas. Ahora se aboga la participación de todas/os en la apreciación, aprendizaje y difusión cultural del patrimonio de los pueblos, grupos y comunidades indígenas. Segundo, asumir que las lenguas indígenas son el primer idioma de los estudiantes no reconoce una heterogeneidad de orígenes histórico-regionales con más de 20 años de asentamiento de las familias indígenas migrantes en el estado de Baja California, quienes tienen un predominio del español como lengua materna. Hay una discriminación etnoracial en las aulas, no hay un diálogo intercultural y sigue pensándose una inferioridad entre las NNA indígenas migrantes en razón de comunicarse en lenguas indígenas (un sentimiento de vergüenza), por el color de piel, las características físicas y el lugar de origen. Estos elementos encuentran una síntesis de la discriminación en el término "oaxaco", "sureño" o "negrito" en la socialización escolar.

Bajo una demanda de justicia e inclusión social en la política migratoria, Garduño, et. al (2010) documentan las prácticas culturales de alrededor de 2 mil familias indígenas migrantes procedentes del estado de Oaxaca que llegan a trabajar al valle de San Quintín, Baja California. Estas prácticas pueden diferenciarse a partir de la pertenencia a la misma etnia, por ejemplo: el grupo cultural zapoteco, el grupo de carnaval mixteco, el grupo cultural náhuatl o el grupo cultural purépecha. O bien, por la pertenencia al mismo pueblo de origen, entre estos: San Juan Mixtepec, San Juan Copala, San Miguel El Grande, San Martín Itunyoso, Chalcatongo o San Agustín Tlacotepec. Estos festivales que se acompañan de música, comida, baile y discursos son un vivo ejemplo de recreación y re-territorialización cultural de las familias indígenas migrantes en Baja California. La presencia de músicos entre las comunidades indígenas migrantes, así como sus condiciones de migración circular a partir de los ciclos de cultivos relacionados con su participación laboral en las huertas privadas y en el comercio de artesanía permiten que la creciente movilización y organización de las familias indígenas en comunidad puedan asumir una ciudadanía, visibilizar las injusticias y conquistar el espacio público. La transformación de la representación social de las familias indígenas migrantes al interior de la sociedad de Baja California, de ser vistos como fuerza de trabajo explotable (y un grupo vulnerable) a ser considerados como agentes sociales portadores de una determinada cultura está en proceso.

Prado (2017) señala que el Programa Nacional de Derechos Humanos (PNDH) para el periodo 2014 a 2018 considera prioritaria la creación de planes y programas para favorecer la protección de los migrantes al reconocerles entre los grupos vulnerables. A la par se ejecutó el Programa Especial de Migración (PEM) debido al rápido crecimiento de la migración en razón a un aumento de 3.2 veces, ya que México representa el principal corredor migratorio del planeta en la búsqueda de ingresar a los Estados Unidos de América (EUA). En este sentido, el PEM se propuso como una política migratoria integral basada en la coordinación interinstitucional desde el discurso de los derechos humanos para la población migrante. La protección de los menores migrantes se ha convertido en una prioridad tanto para el PEM como para la Comisión Nacional de Derechos Humanos. Se pondrá énfasis en los argumentos 
que Prado hace para considerar como no cumplido el derecho a la recreación y el derecho a vivir en una familia que le brinda la seguridad y la protección necesaria para un sano equilibrio en su desarrollo para algunas NNA indígenas migrantes. La condición de la niñez indígena migrante mencionada es muy compleja, la NNA se enfrenta a condiciones de explotación laboral, matrimonio infantil, esclavitud sexual, abuso sexual, reclutamiento por el crimen organizado e incluso tráfico de menores. Pese a que el PNDH de 2014-2018 estipula que los Sistemas Nacional, Estatal y Municipales para el Desarrollo Integral de la Familia (DIF), tienen que habilitar espacios de alojamiento o albergues que cumplan con los estándares mínimos que este señalará para que les sea brindada la atención adecuada a las NNA. Tampoco se ha creado la prometida Procuraduría Federal de Protección de los Derechos de las NNA irregulares, acompañadas o no acompañadas.

Morales (2019) hace mención a "los niños perdidos" tal como Luiselli (1983) llama al desplazamiento forzado de niños centroamericanos y mexicanos indocumentados que cruzan la frontera entre México y E.U.A. para escapar de la pobreza, el abuso sexual y la inseguridad en sus países. Solo entre 2013 y 2014, la Border Patrol (patrulla fronteriza) detuvo a 80 mil menores en territorio estadounidense y esto provocó que se declarará la situación de crisis migratoria infantil de la llamada migración del Triángulo del Norte (Guatemala, El Salvador y Honduras). Los niños perdidos se desplazan por necesidad, por instinto de supervivencia, porque necesitan huir de la realidad que enfrentan en su país. No es una migración cómoda ni segura, es un proceso difícil y doloroso porque ya no se concibe tener que regresar. Estos niños que dialogan con Luiselli dan cuenta de un mosaico de historias de violencia, soledad y desigualdad. Sus voces se escuchan de manera clara y aterradora sobre todo porque se trata de infantes que no buscan trabajo sino protección. En este contexto, la migración aparece como un evento traumático, en los que las NNA migrantes tiene una vida quebrada e incluso durante su tránsito por México algunas/os viven historias de abuso, pornografía, violencia y pobreza iguales o peores que las que presentaban en sus comunidades de origen. Estos desplazamientos tienen un carácter nómada y ocurren en una época donde la globalización y el neoliberalismo asume que las personas migrantes tienen vidas desechables.

Morales (2019) sostiene que el libro de Luiselli tiene un final abierto, sin certeza sobre el futuro de los niños perdidos. El enfoque narrativo-biográfico da importancia a la necesidad de contar estas historias desde las voces, dibujos y representaciones de las NNA migrantes no para arreglar vidas rotas, sino para entender lo que sucede. La migración no es un fenómeno sociológico, sino un evento doloroso, violento y traumático. Bajo este argumento, el lenguaje no puede expresar completamente la dimensión real y subjetiva de la experiencia migratoria de las NAA indígenas en el estado de Chihuahua y en el estado de Sonora. Es común además que quienes cuentan un hecho violento y doloroso se enfrenten al problema de hacer inteligible lo vivido. Se concluye que la única manera de empezar a entender estos años tan oscuros para las NNA será registrar la mayor cantidad de historias individuales posibles, escucharlas una y otra vez, escribirlas una y otra vez para que no sean olvidadas, para que queden en hondo de nuestra conciencia, y regresen, siempre, a perseguirnos en las noches, a llenarnos de espanto y vergüenza (Luiselli, 1983: p. 32, citada en Morales, p. 68). Actualmente, la migración de NNA no acompañados es un problema urgente de atender por las políticas migratorias del país. Al respecto, Gómez (2018) menciona que los artículos 615 de la Ley de Migración de México establecen los siguientes derechos para las y los migrantes extranjeros o nacionales: libertad de tránsito; acceso a los servicios educativos; recibir atención médica urgente para salvar su vida de manera gratuita; autorización de actos de estado civil y expedición de determinadas actas; procuración e impartición de justicia; 
presentación de quejas en derechos humanos; reconocimiento de personalidad jurídica; proporción de información de derechos y obligaciones; derecho a un traductor o intérprete si es necesario; y consideración de discapacidad. En 2016, el gobierno federal capacitó en un nuevo protocolo de actuación a 2,807 funcionarios de 32 delegaciones federales del Instituto Nacional de Migración (INM) y, actualmente, hay más de 15 normas jurídicas, reglamentos y protocolos que protegen los derechos de las NNA migrantes no acompañados en México. Se considera un trato especial en el acceso a servicios que deben brindar los Oficiales de Protección a la Infancia (OPI) del INM.

Desde una visión crítica y anticapitalista, Moctezuma (2018) señala que la política de cero tolerancia a la inmigración de E.U.A., la cual consiste en criminalizar la migración irregular para crear terror como política de Estado del presidente Donald Trump para desalentar la inmigración con base en restricciones en el acceso a los derechos humanos básicos: educación, servicios sanitarios, adquisición de permisos y licencias, permiso de trabajo, etc.; además, la ausencia en la suscripción de las Convenciones Internacionales sobre derechos humanos respecto de los menores migrantes irregulares, acompañados o no acompañados. A esto se añade, la omisión del gobierno federal mexicano del presidente Enrique Peña Nieto con diferentes obstáculos para la creación de una fiscalía a favor de los derechos de las NNA, que entre otras cosas indague, atienda y evite que los menores se vuelvan a convertir en víctimas de abusos psicológicos, sexuales, laborales y sociales. Ambas políticas atentaron contra la dignidad humana de las NNA migrantes desde 2013 y hasta la fecha actual. De octubre 2017 a julio 2018 había un total de 36,390 menores migrantes no acompañados detenidos por la Border Patrol y la mayoría tenía entre 13 a 17 años (p. 138).

Habrá que reconocer que alrededor de la mitad de las NNA migrantes no acompañados se desplazan solos debido al abuso sexual, la violencia doméstica, la orfandad o un contexto social violento en el que la repatriación humanitaria, la reunificación familiar y el regreso al hogar es imposible y no deseado. Esta situación es omitida en los centros de detención de la Border Patrol, en los albergues estadounidenses Shelter for Minors y en los albergues mexicanos del DIF donde las NNA migrantes en tránsito reciben algunos apoyos y pocas veces son escuchadas/os. Los albergues más grandes se encuentran en Nogales, Son.; Tijuana, B.C. y Ciudad Juárez, Chih. Además, en años de alta demanda, las juventudes migrantes con condiciones óptimas de salud y con rasgos de personalidad específicos (funcionales) son seleccionados como fuerza de trabajo para el capital estadounidense (Moctezuma, 2018).

Con el cambio en la gestión presidencial de 2018, el gobierno federal dirigido por el presidente Andrés Manuel López Obrador ha mantenido una posición humanitaria y no restrictiva hacia la migración. Lo cual ha traído una modificación en las prácticas y políticas migratorias, por mencionar algunas: garantizar los derechos humanos de migrantes; concebir la deportación como el último recurso; combatir discurso discriminatorio y xenofóbico contra los inmigrantes; abordar el fenómeno de migración como la consecuencia de un modelo económico global que ha agravado la desigualdad entre los países subdesarrollados y las potencias mundiales; crear un fideicomiso de alerta humanitaria destinado a los municipios fronterizos a los que ha llegado la caravana migrante. Apoyar en particular a las NNA, se estima una presencia en el territorio mexicano de más de 2,300 NNA provenientes de las caravanas migrantes que salieron el sábado 12 de octubre de 2018 de San Pedro de Sula, Honduras. Quienes se han unido para huir de la violencia de sus países y tratar de llegar a EUA para pedir refugio, y así, buscar una mejor calidad de vida (Gómez, 2018). 


\section{Planteamiento epistemológico y metodológico}

La pregunta de investigación que se planteó con la realización del trabajo fue: ¿cuál es la situación de vida en relación con el derecho al juego, el acceso a la educación y los cuidados de las niñas, niños y adolescentes (NNA) indígenas migrantes en las colonias emergentes de la región noroeste del estado de Chihuahua y la región noreste del estado de Sonora? En consecuencia, se recurrió al método biográfico descrito por Sautú (1999) para presentar experiencias de las NNA indígenas migrantes y sus familias bajo el paradigma interpretativocomprensivo con una perspectiva de género. Para efectos de esta exposición, se recuperan únicamente los resultados de los relatos temáticos de vida realizados entre 2013 a 2016 en la región serrana limítrofe entre Chihuahua y Sonora. Los resultados se presentan hasta ahora en 2019 luego de que el autor tiene la posibilidad y la seguridad de difundir el proyecto de investigación que se realizó con múltiples obstáculos debido a una metodología de alto riesgo iniciada en el contexto de la Guerra contra el Narcotráfico de 2007-2012 por el presidente de los Estados Unidos Mexicanos, Lic. Felipe Calderón Hinojosa. El temor principal del grupo surge de amenazas recibidas por dueños de Huertas Privadas por denunciar maltratos y explotación laboral infantil, así como por un llamado hecho por colegas criminólogos a no trabajar el tema por el descubrimiento de fosas con menores desaparecidos (asesinados) y que han sido ocultas por los tres niveles de gobierno.

De la O y Flores (2012) estudian a través de entrevistas las condiciones de violencia que viven las juventudes en la frontera norte de México. Señalan que el reclutamiento forzado de adolescentes y jóvenes por las organizaciones criminales se apoya en una política de miedo en la sociedad de Matamoros, Tamaulipas. En medio de esta guerra, destaca el problema de la imprecisión de las fuentes oficiales, que no permiten conocer con certeza el número de niños y niñas detenidos, heridos, asesinados o desaparecidos en los últimos años.

Los medios de comunicación y las organizaciones sociales ofrecen números aproximados de sus propios conteos sobre la niñez, pero, estos y las fuentes oficiales no coinciden. La Secretaría de la Defensa Nacional reportó 232 niños y niñas retenidos en operativos militares contra la delincuencia organizada entre 2007 y mayo de 2010 en varios estados del país, entre los que sobresalen Baja California, Veracruz y Guerrero. La Secretaría de Marina (Semar) reportó sólo 26 apresados en el mismo periodo, y la Policía Federal registró 363 niños y niñas detenidos durante operativos contra el crimen organizado, de los que 148 fueron aprehendidos en Chihuahua, entre 2008 y mayo de 2010. La Procuraduría General de la República ubica en 3,664 la cifra de niños y niñas detenidos vinculados con la delincuencia organizada entre diciembre de 2006 y abril de 2010. El año con más detenciones fue 2009 (REDIM, 2011, Pp. 38-39, citado en De la O y Flores, 2012). Los homicidios y las lesiones relacionadas con el crimen organizado, sobre la infancia y adolescencia y por la infancia y la adolescencia, son un hecho cotidiano en el país: niños y jóvenes empleados o que participan de cualquier manera en la violencia armada organizada donde hay elementos de una estructura de mando y dominación sobre un territorio, su población local o sus recursos (Downey, 2005: 9, citado por REDIM, 2011, p. 36, citado en De la O y Flores, 2012).

Se propone la aplicación de la fenomenología, la etnografía y el método narrativo-biográfico como diseño metodológico combinado para obtener la información necesaria desde la entrevista, la observación, la narrativa y el análisis descriptivo de la situación de vida de las NNA indígenas migrantes en las colonias de reciente creación Ejidos o Secciones localizadas en los municipios de: Bacerac, Bavispe y Agua Prieta, Son. y Casas Grandes, Janos y Ascensión, Chih. Fue necesaria una triangulación en el uso del dibujo infantil como 
instrumento para recolectar información de forma (no)verbal y la participación del grupo de investigación en juegos de las NNA indígenas migrantes para contextualizar las opiniones, recuerdos, metáforas o incluso creencias que tienen sobre el juego infantil, las experiencias educativas, las tareas o labores femeniles y masculinas. Al respecto, Ander-Egg (1995) y Arias (1981) considera que es necesaria la combinación de métodos para poder reconstruir las condiciones de vida de los sujetos de estudio en contextos al límite como es el caso de las NNA indígenas migrantes.

Aguirre y Jaramillo (2012) consideran que la fenomenología y su método contribuyen, de modo privilegiado, al conocimiento de las realidades escolares desde la descripción y las vivencias de los sujetos estudios. La fenomenología requiere arduo estudio y dedicación, ya que la rigurosidad exige considerar que el método fenomenológico consistirá en partir de la actitud natural con mayor interés por el análisis reflexivo sobre la relación sobre las experiencias de vida y la condición de vida marcada por el contexto sociohistórico y cultural. Ante esto, se parte de la epojé de la reducción eidética y de la epojé de la reducción trascendental para llegar a la constitución de la esencia del fenómeno de estudio (eidos). Esta reconducción se realizará mediante las unidades de sentido de las vivencias referidas durante las entrevistas entre el investigador y los sujetos de estudio con apoyo de preguntas o temas con relación a la problemática abordada. La fenomenología permite ser más conscientes de que con las narraciones es posible dar sentido y significado de una realidad marcada por el mundo de la vida, es decir, el valor de las personas, sus experiencias subjetivas frente a los sedimentos históricos que determinan a la vez su condición de vida.

Martínez (1999) define las investigaciones etnográficas, es decir, aquellas que tratan de describir e interpretar las modalidades de vida de los grupos de personas habituadas a vivir juntas. El enfoque etnográfico se apoya en la convicción de que las tradiciones, roles, valores $\mathrm{y}$ normas del ambiente en que se vive se van internalizando poco a poco y generan regularidades que pueden explicar la conducta individual y de grupo en forma adecuada. En efecto, los miembros de un grupo étnico, cultural o situacional comparten una estructura lógica o de razonamiento que, por lo general, no es explícito, pero que se manifiesta en diferentes aspectos de su vida. En este sentido, el investigador etnográfico, al desear acercarse a la verdadera naturaleza de las realidades humanas, se centra en la descripción y la comprensión.

Se recurre a la mirada infantil sobre las relaciones laborales y la condición del cuidado desde las voces de las NNA indígenas migrantes en ambas regiones en la región limítrofe entre Chihuahua y Sonora. Sin duda, los estudios sobre el mercado de trabajo se realizan comúnmente desde el enfoque de género, la cohorte generacional, las categorías ocupacionales y el tipo de centro de trabajo. Se delimitan los sujetos de estudio, por ejemplo: grupos de hombres y mujeres; grupos identificados ideológicamente con algún cambio político o movimiento social; grupos diferenciados por el puesto de trabajo; grupos afiliados al sector público o sector privado; entre otros más. Sin embargo, la condición de vida de las NNA indígenas migrantes como sujetos de estudio está marcada por la violación a los derechos humanos (restricciones a la población migrante para trabajar en empleos formales), injusticias (políticas migratorias represivas con interés en la repatriación), una interseccionalidad de género (discriminación con base en la diferencia sexual, el grupo etario, la pertenencia indígena, el estatus socio-económico, la situación migratoria, la ciudadanía de $\mathrm{n}^{\prime}$ número de orden, la historia de inferioridad y los factores de riesgo asociados con la vulnerabilidad). 
El documento analiza los posicionamientos de las NNA indígenas migrantes en Chihuahua y en Sonora, su relación con las políticas migratorias existentes y el proceso de construcción de su identidad con respecto a la ausencia de derechos, la victimización vía su sujeción a los grupos vulnerables y la no actuación pública frente a la explotación laboral infantil en huertas privadas, el reclutamiento de las organizaciones del crimen organizado y la violencia doméstica (incluido el abuso sexual por parte de algún familiar). Hablar de las políticas de cuidado de las NNA indígenas migrantes implica reconocer que su condición de vida es determinada por el modelo económico capitalista, así como las creencias que se aceptan socialmente sobre la población indígena (como grupo vulnerable, la victimización y la vida al amparo de la sociedad anfitriona), que fundamentan la discriminación etnoracial e incluso que tienen su origen en las decisiones del padre y la madre del menor, entre estas: asociadas a la colecta de propinas o ayudas, el trabajo infantil como complementación del gasto familiar, la trata de niñas/os con grupos delincuenciales y entrega de niñas a hombres con fines matrimoniales o de pornografía infantil. Bajo estas condiciones, las NNA indígenas migrantes tiene pocas oportunidades para ejercer su derecho al juego y el derecho a la vida libre de violencias, la dignidad, la recreación, la educación y el trabajo digno.

Cabe señalar que cuando se delimitó el objeto de estudio se pudieron identificar que hay dos tipos de grupos indígenas migrantes o de tránsito entre Chihuahua y Sonora. El primer grupo corresponde con los comerciantes -en su mayoría hombres- de artículos para el hogar que viajan en camiones de carga y trabajan bajo condiciones laborales al límite -reciben violencia (intento de homicidio), hostigamiento, secuestro, extorción, robo o discriminación por grupos criminales y por las propias autoridades municipales en toda la región analizada- y vulnerabilidad social - no cuentan con acceso a los sistemas públicos de salud y duermen en los camiones estacionados en las gasolineras, e incluso durante el invierno entierran sus cuerpos en la tierra para protegerse del frío-. El segundo grupo se conforma por las familias indígenas migrantes procedentes de otras entidades federativas: Chiapas, Oaxaca, Guerrero y Veracruz, que vienen exclusivamente a trabajar en las huertas privadas de la región Chihuahua-Sonora-Sinaloa-Baja California. Para la mayoría de las familias en ambos grupos no existen derechos laborales, civiles, de salud, recreativos, culturales ni educativos.

\section{Contexto de las familias indígenas migrantes en el estado de Chihuahua}

Para 2014, el país contaba con 120 millones de habitantes, 11.9 por ciento de estos pertenecen a algún grupo indígena y 7.1 por ciento son hablantes de lengua indígena (HLI), por lo que uno de cada diez mexicanas/os son indígenas. Cabe mencionar que 73.2 por ciento de ellas/os viven en condición de pobreza -alta y muy alta marginación - frente a 43.2 por ciento de la población no indígena. Respecto al derecho a la educación, las niñas/os indígenas tienen menor asistencia escolar que se agrava durante el tránsito del nivel de educación preescolar hasta el nivel de educación superior $-1 / 4$ de las/os HLI son analfabetas-, 31.4 por ciento reprobaron uno o más grados escolares, 9.5 por ciento tienen una situación de extra-edad, alrededor del 50 por ciento tuvieron un desempeño por debajo del nivel básico aceptable en las pruebas estandarizadas de español y matemáticas en primaria, 77.1 por ciento cuentan son becarios de programas de asistencia social federales, 24.4 por ciento de las madres cuentan con estudios de secundaria, 31.9 por ciento reciben apoyo familiar para la realización de las tareas escolares, 11.2 por ciento cuenta con acceso a Internet en el hogar, 58.5 por ciento realizan labores domésticas y 18.1 por ciento trabaja fuera de casa. Respecto al personal docente, sólo se sabe que 60 por ciento de los docentes noveles del programa de 
licenciatura en educación intercultural bilingüe son mujeres, 67 por ciento agradece la oportunidad de acceso a la educación superior y 68 por ciento de ellas tuvieron un logro insuficiente en los exámenes generales de conocimiento al egresar de la educación superior (INEE, 2015).

En el estado de Chihuahua, la Coordinación Estatal de la Tarahumara (denominado como CET, 2010) estima que la población indígena en la entidad es aproximadamente de 120000 personas, de las cuales 90 por ciento son tarahumaras o rarámuris, ocho por ciento son tepehuanos u ódamis, uno por ciento son guarojíos o makurawe y uno por ciento restantes pertenecen al pueblo pima u o'oba. Además, por sus características geográficas y productivas, la región serrana se divide en dos zonas; alta tarahumara, que se integra por 14 municipios, ubicados en área de bosque y que concentran 76 por ciento de la población de la zona; y la baja tarahumara, conformada por nueve municipios en los cuales predominan las barrancas y ahí se ubica el 24 por ciento restante de la población.

Por otro lado, los asentamientos de indígenas migrantes se dan en las diferentes ciudades del estado de Chihuahua, donde las familias han construido colonias emergentes en las últimas tres décadas. Asimismo, las familias indígenas migrantes pertenecen a diversas comunidades de los pueblos rarámuri, ódame, guarojía y o’oba, así como a comunidades procedentes de otras entidades federativas que se reconocen por su lengua: náhuatl, mixteco, mazahua, zapoteco, tlapaneco, otomí, triquis, huicholes, entre otros. Las comunidades más numerosas de familias indígenas migrantes que han llegado de otras entidades del país, son: mazahuas, mixtecos, zapotecos, triquis y huicholes. Se estima que existen aproximadamente 16,000 indígenas migrantes en Ciudad Juárez y 15,000 más en las ciudades de Chihuahua, Cuauhtémoc y Delicias (CET, 2010).

La pobreza es un concepto complejo de entender cuando se habla de la comunidad indígena, ya que hay que considerar diversos aspectos históricos y estructurales que han situado al indígena en condiciones de desigualdad, desventaja y estigmatización con respecto a otros sectores de la población (CET, 2010). Desde la perspectiva del desarrollo humano la pobreza queda relacionada a la desprotección respecto al derecho de salud, educación, vivienda, empleo y vida libre de violencias, en sus contextos de equidad, justicia y dignidad. Hay que reconocer que se han realizado esfuerzos institucionales para mejorar las condiciones de vida de las familias indígenas, empero, es necesario redoblar el trabajo público y de las organizaciones civiles para responder con mayor fortaleza a las necesidades desde las instituciones de educación y salud, el espacio comunitario y la procuración de justicia.

Cabe mencionar que las principales causas de mortalidad en la población indígena en el estado de Chihuahua, son: La tuberculosis, las infecciones gastrointestinales en verano e infecciones en vías respiratorias en el invierno; otra causa de mortalidad es la desnutrición, principalmente, en niños menores de cinco años y mujeres en gestación (CET, 2010).

Además, el estado de Chihuahua es una entidad con una gran diversidad cultural, empero, gobernada por políticos que conciben a la sociedad chihuahuense como un grupo social homogéneo, incluso, los silencios generados por el régimen despótico y autoritario del gobierno estatal sobre las violencias contra las minorías se mantienen ocultas desde el siglo XVIII. Chihuahua es el estado más grande del país y también es el más vacío de derechos humanos -constantemente se refuerza el discurso de inexistencia de raíces africanasindígenas, feminicidio, crimen organizado, segregación, despojo de tierras y corrupción pública-. La situación de vida de la niñez indígena migrante lleva consigo una vulnerabilidad frente a la cultura patriarcal, la perversión masculina y la exclusión del indígena del sistema educativo y de salud pública. 
La educación pública en Chihuahua no puede inscribirse como un proceso para la construcción de una educación intercultural bilingüe o una coeducación en la que participen todos sin distinción de origen, raza, sexo, ideología, religión, edad o condición social. La sierra limítrofe entre Chihuahua y Sonora ha sido la región menos beneficiada por los recursos públicos. Por otro lado, el estado de Chihuahua, tiene un panorama estadístico pesimista de la población indígena sin ningún grado escolar: 54.4 por ciento para las mujeres y 39.4 por ciento para los hombres nunca culminaron los primeros años de escolaridad. Mientras que para la población en general son inferiores a seis por ciento (CET, 2010).

Cabe señalar que los jóvenes que provienen de grupos marginados se enfrentan a serios obstáculos para tener acceso a las Instituciones de Educación Superior (IES), permanecer en éstas y graduarse oportunamente. Mientras que 45 por ciento de jóvenes en el grupo de edad entre 19 y 23 años, que viven en Chihuahua capital pertenece a familias con ingresos medios altos. La capital del estado recibe educación con mayor calidad. Habrá que añadir que la tendencia ha sido llevar a las comunidades más pobres del estado Universidades Tecnológicas y Universidades Privadas con una calidad educativa igualmente pobre, por lo cual, la población en la región noroeste de Chihuahua y en la región noreste de Sonora realiza sus estudios en IES donde se asiste para obtener el título de licenciatura, los jóvenes carecen de aulas con discusiones académicas relevantes y con asesorías de calidad -los encargados de las IES son priistas y trabajan para el partido, no para el desarrollo científico, tecnológico y cultural de las comunidades-.

Por otro lado, el asunto de la vivienda desde el concepto de los pueblos y comunidades indígenas, significa algo más que tener una casa, es el entorno, las rocas, el arroyo, los pinos y el patio. Esto forma una parte del contorno en el que se constituye el carácter de las familias indígenas. En el año 2000, según la información censal existían en el estado de Chihuahua 755.379 viviendas particulares, de las cuales 27,967 familias eran representadas por una jefatura de hogar con pertenencia indígena, cifra que representa 3.7 por ciento del total. El porcentaje de viviendas indígenas con un cuarto es de 43 por ciento, por lo que existe un alto índice de hacinamiento, sobre todo si se considera que el promedio de ocupantes de estas viviendas es de cinco personas o más.

En lo que se refiere a los materiales de construcción, en las viviendas indígenas, el que predomina en las paredes es el adobe, el cartón negro y la madera; los materiales sólidos se encuentran en un 30 por ciento de las viviendas. Con referencia a los pisos de las viviendas, de cada 100 viviendas 58 tienen piso de tierra, entre los materiales de recubrimiento utilizados están el cemento y la madera. Los techos de las viviendas son construidos con lámina de asbesto o metálica en un 39.3 por ciento, los de palma tejamanil o madera alcanzan 34.1 por ciento y el resto con otros materiales -cartón y forro de plástico-. En lo referente a los servicios de la vivienda, 95 por ciento de las viviendas indígenas de la Sierra Tarahumara no cuentan con energía eléctrica, 92 por ciento carece de agua entubada y 99 por ciento no dispone de drenaje y alcantarillado (CET, 2010). En este mismo contexto están las escuelas de la región.

En cuanto a la Ley de la Coordinación Estatal de la Tarahumara, creada en 1987, establece artículos centrados en: 1. Bienestar de los grupos indígenas. 2. Desarrollo socio-económico de la región tarahumara. 3. Otros relacionados al sistema de organización de la comunidad tarahumara. Por lo cual existe una ausencia jurídica en el trato de las necesidades culturales, laborales y de seguridad humana de la población indígena tarahumara. Al respecto, la ONU pone a los tarahumaras como los más desprotegidos de México. Desde 2008 hace una alerta de abandono. En el año 2012 se menciona que la comunidad tarahumara es la etnia más 
desprotegida y marginada del país en el Informe sobre Desarrollo Humano de los Pueblos Indígenas en México.

El Diario Norte de Ciudad Juárez reporta que el municipio con menor avance es Batopilas, ubicado en el corazón del estado de Chihuahua, de acuerdo con el Índice de Desarrollo Humano de la Población Indígena (IDH-PI). Esta región de Chihuahua reportó un indicador de 0.3010 , cifra mucho más baja que el país con menor desarrollo humano en el mundo, que es Nigeria, con un índice de 0.33 . Además, 40 por ciento de los niños indígenas padecen desnutrición infantil y en enfermedades respiratorias de carácter crónico, cifra tres veces mayor a la registrada en niños no indígenas o mestizos. Dicha situación es preocupante no sólo por los efectos que tiene sobre el bienestar de una generación, sino porque se transmite de padres a hijos, provocando un círculo de pobreza y desigualdad de oportunidades que condena a la población en desventaja y a sus descendientes a permanecer en las mismas circunstancias.

\section{Resultados}

El municipio de Ascensión está situado al norte del estado de Chihuahua, en la frontera con Estados Unidos, en particular con el estado de Nuevo México, donde limita con el Condado de Luna, el Condado de Doña Ana y el Condado de Hidalgo. Dentro del territorio mexicano, el municipio de Ascensión limita al oeste con el municipio de Janos, al este con el municipio de Juárez, al sureste con el municipio de Ahumada, además, al sur con los municipios de Buenaventura y Nuevo Casas Grandes; tiene una extensión territorial de $11,000.10 \mathrm{~km}^{2}$ que constituye el 4.45 por ciento de la superficie del estado, es el cuarto municipio más grande del estado y el 17 más grande del país.

La localidad de Ascensión está situada en el municipio de Ascensión, cuenta con 10,961 habitantes y está a 1300 metros de altitud, por lo que hay un clima propicio para huertas de cultivo. Dentro de este municipio se encuentran diversas familias indígenas migrantes procedentes del sur del país, los cuales emigran de las entidades de Oaxaca y Guerrero, la mayoría de las familias vienen a trabajar a este municipio en las huertas privadas como jornaleros. En esta labor participan todos los miembros de las familias y es de esta manera como reúnen el sueldo de la semana para solventar sus gastos.

El trabajo en las huertas privadas es de tipo informal, de contratación inestable, sin prestaciones públicas, con ingresos irregulares y de jornadas extensas. Esta situación se ha naturalizado entre las familias indígenas migrantes en el estado de Chihuahua, quienes descuidan elementos necesarios para el mejor desempeño laboral y relaciones familiares humanas, como: la apariencia, la nutrición, la higiene personal, la salud física, la cooperación, la solidaridad, el amor y el afecto. Por ejemplo, la exposición directa y prolongada a los rayos del sol, la alimentación basada en tortilla, chile y frijoles, la conducta sexual de riesgo, el trabajo con animales de granja sin requerimientos sanitarios, la violencia familiar, el abandono del cuidado infantil, entre otros.

Fotografía 1. Trayecto de las familias indígenas migrantes desde las hurtas privadas 


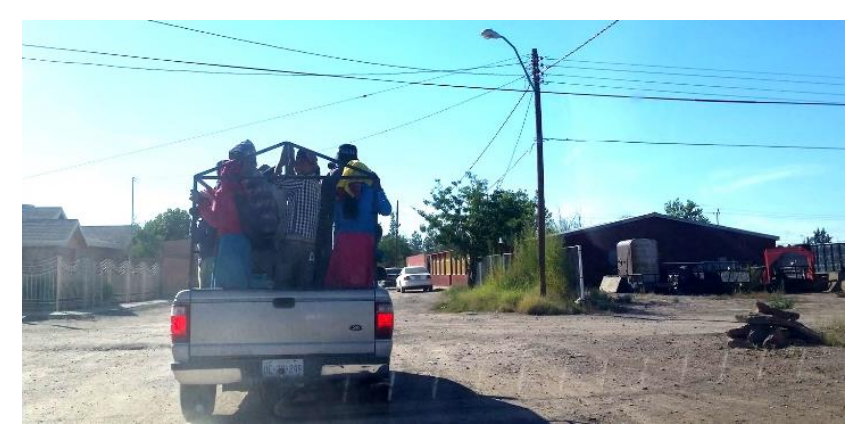

Fuente: Fotografía tomada durante la etnografía en Ascensión, Chih.

La lengua nativa de las familias indígenas migrantes es el mixteco, el náhuatl y el tlapaneco, aunque tienen en promedio nueve años residiendo en el estado de Chihuahua algunos de los miembros tienen dificultades para leer, escribir, hablar y entender el idioma español. Todas las familias entienden la lengua mixteca ya que saber este dialecto es parte de una táctica para sobrevivir en el mercado laboral de la región. Al respecto, la contratación de las familias indígenas migrantes en las huertas privadas de la región noroeste del estado de Chihuahua se realiza de manera informal y el trabajo se considera inestable o temporal, la población indígena activa no tiene prestaciones médicas pese al cumplimiento de jornadas de trabajo mayores a ocho horas diarias, así como a los problemas que derivan de trabajar bajo los rayos del sol o en climas extremosos. La situación laboral se dificulta en la medida en que la mayoría de la población indígena no aprende a leer, escribir y hablar en español.

Los problemas más fuertes en que incurren las familias indígenas migrantes es la trata de mujeres menores de edad a cambio de la dote de bienes muebles e inmuebles. La recurrente muerte del producto durante el embarazo, incluso, la muerte infantil por accidente o descuido. También se presenta el regalo o la venta de niñas/os y bebés a otras familias, muchas veces este es el motivo por el cual deciden no registrar a sus hijas/os.

Se pone el caso de O. G. quien viene de Guerrero y tiene más de dos años viviendo en Ascensión, él vive de su trabajo como jornalero, habita en casa de su prima I. R. de 22 años de edad quien tiene ya tres años en este municipio. En casa de I. R. también vive su hermana L. R. de 17 años de edad, las dos trabajan como jornaleras, I. R. se quedó esta temporada aquí, ya que su mamá y su hermana mayor A. R. de 25 años de edad están en Hermosillo, estado de Sonora trabajando.

O. G. llegó al municipio a trabajar, en este último año trajo a su esposa J. L. y a su hijo D. O., el matrimonio ya tiene dos hijos, uno de siete años llamado D. O. y un bebé que recién nació que se llama R. G., D. O. no acude a la escuela primaria porque cuida a su hermano mientras ambos padres están todo el día en la huerta. No cuentan con ningún servicio médico, los niños andan descalzos por la casa cuentan con dos cuartos donde viven todos -incluyendo a I. R. y L. R.--, el terreno es pequeño. Este año, pese a que trabajaron todos los días durante ocho horas, el pago fue poco y no alcanzaron a ahorrar para pasar la temporada desocupada entre diciembre-febrero. La familia se vio obligada a irse a Agua Prieta, Son., luego a Hermosillo, Son., y después a Culiacán, Sin., para sobrevivir junto a otros indígenas migrantes que radican allá. En este hogar son cuatro personas adultas las que trabajan, en ocasiones D. O. trabaja en la huerta para ayudar con los gastos de la casa. Ambos niños muestran baja estatura, manchas en la cara y falta de higiene. Estas cuatro personas cuentan con un sueldo aproximado de 600 pesos por semana, es así como logran solventar los gastos. 
Otra familia es la familia Campos, ellos son originarios de Tlalpa, Guerrero, todos hablan tlapaneco, uno de los integrantes es el señor R. C. quien está casado con la señora C. S. El matrimonio tiene dos hijos, M. C. de 21 años de edad casado y con dos hijos de cuatro y tres años. El otro hijo del matrimonio es J. C. de 19 años, ambos hermanos, padres y esposa de M. C. trabajan en la pisca del chile. También, en esta casa vive la mamá del señor R. C. con 62 años de edad, se llama E. M. La casa la comparten con el señor M. A. de 33 años de edad casado con la señora A. C., los cuales tienen cuatro hijos: A. A. de ocho años de edad, D. A. de seis años de edad, M. A. de cuatro años de edad y M. A. de dos años, de los cuales sólo A. A. va a la escuela por la tarde y en las mañanas todos trabajan en las huertas. En la casa también viven S. C. 18 años de edad y F. E. de 22 años, los cuales tienen un hijo llamado N. E. de dos años de edad.

Otra de las familias que viven en este municipio está conformada por el señor F. G. y la señora C. S., quienes tienen ocho años radicando en esta localidad, los cuales tienen cinco hijos: la mayor M. G. de 20 años de edad quien trabaja en el campo como jornalera y tiene una hija M. R. de dos años que está aprendiendo el trabajo en la huerta, I. R. de 15 años de edad que por las tardes estudia tercero de primaria, P. R. de 12 años de edad que está en segundo grado, A. R. de nueve años de edad que no va a la escuela, S. R. de cinco años que aún no está registrada. Todos hablan español y tlapaneco, cuentan con seguro popular porque el señor F. G. es capataz, tienen casa propia con tres cuartos, con todos los servicios: agua, luz, gas de tanque y piso de cemento. La familia está establecida ya en el municipio, ahorran para la época en la que no pueden trabajar para sobrevivir de sus ahorros y no tener que migrar. No tienen planes de regresarse a su tierra, quizá un día solo quieren ir de visita.

Otro caso es la familia de P. C., quien llega en mayo y se va a Sinaloa en diciembre. Ella viaja con su esposo e hijos, así como dos nueras. Sus hijos son G. N. de 22 años de edad quien está casado con L. S. de 17 años y tienen un bebe de un año. Su otro hijo es E. N. de 17 años, casado con E. L. de 16 años. En la casa también viven algunas niñas, niños y adolescentes indígenas migrantes no acompañados que se quedaron solos en México porque sus padres emigraron hacia Estados Unidos de América (E.U.A.), entre estos: B. R. de 13 años que trabaja en la huerta, R. R. de 10 años de edad quien va a la escuela por las tardes, igual que J. F. de ocho años de edad y, por último, I. A. de seis años de edad quien aún no va a la escuela. Ellos se van junto con la familia de P.C. de diciembre a marzo a Culiacán, Sin., y después regresan a Ascensión para trabajar. En Sinaloa, el salario es mejor, por lo cual, las niñas/os no trabajan y pueden dedicarse a la escuela. En Sinaloa, se disfruta mejor que en Chihuahua porque allá pueden participar con sus tradiciones en el Carnaval, algunos de ellos tocan la guitarra y las niñas cantan en los eventos.

Desde la etnografía de la infancia, se reconoce que la visión emic de las NNA indígenas migrantes se asume desde tres diferencias: 1. La niñez menor a tres años se considera improductiva, demanda cuidados y aunque no tienen juguetes realizan algunos juegos necesarios para su desarrollo; 2. La niñez mayor de tres años y menor a seis años participa en las labores agrícolas junto a los hermanos mayores y sus padres cumpliendo la jornada completa; 3. Las NNA entre siete y 17 años participan en las labores y sus pláticas implican relación con el crimen organizado y juegos sexuales en los que sus padres no se meten, quizá porque implica una prostitución o explotación infantil evidente por los contenidos de sus pláticas.

Respecto al primer grupo se platicó y observó desde afuera de las casas a diferentes niñas y niños solos que esperaban a que sus padres regresaran de la jornada laboral, quienes sintieron 
confianza con el grupo de investigación. Del registro de observación e impresión podemos citar lo siguiente:

“...) afuera de la casa había un niño de dos años cuidando a un recién nacido, quizá con cuatro meses, el niño tenía al bebé en el suelo, él parecía haber sido obligado a no separarse del bebé o bien a saber que debía permanecer atento al mismo. El niño no supo decir su nombre y, pese a que no estaba en la etapa locutiva del lenguaje, pudimos apreciar que él mostraba con frecuencia objetos al bebé y le explicaba qué eran o para qué eran (piedras, tierra, un pedazo de madera, tortillas y frijoles que estaba limpiando para quitarles las piedras). Tenía al lado un sartén con agua y frijoles batidos, vimos como compartió el agua con el bebé para calmarlo cuando este lloraba, tomaba el agua con un vaso y le daba pequeños sorbos al bebé”. El niño tenía bajo peso, pero, no tenía retraso madurativo, pues, como ya se dijo cumplía con tareas de cuidado de un bebé y demostraba interés por enseñarle a conocer los objetos de su contexto".

Entre los juegos del segundo grupo, se describen los siguientes comportamientos:

....) dos niñas de seis y ocho años se encontraban jugando con un hermano más grande (12 años), ellas corrían detrás de él hasta alcanzarlo, parecía que luego él iba a correr detrás de ellas, pero, no era un juego. Platicaban con él mientras trataban de alcanzarlo, lo hacían y, entonces, él seguía la plática mientras nuevamente las niñas volvían a correr tras él. Después, el hermano mayor salió de la casa para irse con su padre y las niñas se quedaron sentadas. Comenzaron a jugar a decir cómo sería para ellas ser madres y cuántos hijos querían tener. Luego, les pedimos que nos enseñaran algunas palabras náhualt y dijeron que si podían porque les gustaban cómo sonaban, nos enseñaron a decir: nantli (mamá), tahtli (papá) y icniuhtli (hermano), luego, aprovecharon un hormiguero para llevarnos hasta él y decirnos que las hormigas eran azcatl y yo les pregunté por un perro y me dijeron que itzcuintli. Después, volvimos a la casa y nos enseñaron una canción infantil, todos juntos cantamos mientras simulábamos cargar con nuestros brazos un bebé y también ellas nos enseñaron gestos de amor y cariño hacia el supuesto bebé: manocoxteca noxocoyotzin, manocoxteca nopitelontzin, macochi cochi pitentzin, macochi pitentzin, manocoxteca pitelontzin, macochi cochi noxocoyotl".

Respecto a las pláticas del tercer grupo, se recuperan las siguientes conversaciones:

“...) bien que viste como el hombre la trajo tarde, dime si te dio más dinero, no te vayas a preñar pendeja“ (una adolescente de 13 años que ejerce la prostitución con hombres).

“(...) ya ves que él quiere que otra vez lleve las armas para cuidarlo“ (nos permitió tomarle una fotografía que aparece al final del artículo).

“...) voy a espiar a los morros que trabajan allá en W“* (nos dio información de la casa y lugar que se omite por seguridad).

.(...) la vez pasada yo (un niño de 14 años) y mi primo de 16 años llegamos de quebrarnos -asesinar- unos putos en Bacerac y estuvimos varios días moviéndonos en taxi y comiendo chido -sabroso o rico-".

....) no soy sicario, pero, si he tenido que disparar, son cosas de las que no puedes decir que no y no puedes hablar porque he visto como le dan madrazos a los que ya no quieren ir" (un niño de 10 años nos comentaba sobre el reclutamiento forzado por los narcos). 
“(...) no me gusta, pero, está bien, si me porto bien con ellos me dan más dinero“ (una adolescente de 13 años nos comenta sobre cómo se deja tocar y tener relaciones con varios hombres).

....) nosotras ya no somos indígenas, nosotras ya vivimos en una casa y vamos a la escuela, tenemos otra vida" (el conflicto de identidad visible en una estudiante de preparatoria que pertenece a una familia indígena migrante procedente de Guerrero que se asentó en la ciudad desde hace ocho años).

La educación es un proceso cultural, por lo que las NNA aprenden a actuar según las exigencias del contexto social. En Ascensión, Chih., las NNA indígenas migrantes no cuentan con tiempo para el juego ni tampoco para ir a la escuela, ya que tiene que insertarse en el trabajo en huertas privadas. La intención del autor es reconocer las actividades recreativas y valorar los juegos de las niñas/os indígenas migrantes en el municipio de Ascensión, Chih. Destaca por un lado el jugar a los carritos y a las muñecas. Para la observación se consideró el comportamiento de las NNA, las edades, el nivel de solidaridad e incorporación a la vida social, las dinámicas de juego, el tiempo empleado en cada juego y las características del lugar en que se realizó el juego.

La mayoría de las niñas/os que juegan durante más tiempo son menores de siete años, ellas/os, la mayoría habla español. Es difícil que las niñas/os asistan a las escuelas puesto que el trabajo en huertas es inestable y tienen que migrar a otras ciudades para sobrevivir. Para los responsables de familia, el cuidado y la infancia que han tenido sus hijas/os no ha sido buena, no como la que ellas/os tuvieron cuando eran niñas/os y jugaban en sus comunidades en Oaxaca, por ejemplo: las niñas/os indígenas migrantes en Ascensión, Chih., por lo general realizan juegos que terminan en peleas e incluyen agresiones verbales. Las niñas juegan a perseguirse y a las muñecas. Mientras que los niños juegan a explorar el barrio, hablar de cosas de adultos y pelear de mentiras para probar su fuerza. Las madres de las niñas/os frecuentemente tiene pocas palabras para expresarse -sus respuestas a preguntas realizadas por los autores eran pos'sí o pos'no (pues sí o pues no)-, la mayoría de ellas tienen que trabajar y dejan a la hija mayor al cuidado de las y los pequeños.

La mayoría de las familias indígenas migrantes se dedican a la agricultura y fruticultura. La mayoría de las huertas privadas contratan mano de obra indígena porque es más barato y porque la población migrante tiene pocas posibilidades de demandar prestaciones sociales o expresar sus demandas, además, porque son protegidos por las autoridades locales. La mayoría de las NNA realizan actividades vinculadas con la cosecha del chile, la papa, la cebolla, la sandía y el frijol. El autor considera que el DIF en los municipios de la sierra limítrofe entre Chihuahua y Sonora atiende a menos de 230 NNA indígenas migrantes, menos de una sexta parte del total de familias indígenas que transitan por los municipios considerados. De estos, en Ascensión solo 13 NNA reciben apoyos para continuar sus estudios de secundaria o preparatoria. La mayoría de las NNA indígenas migrantes no acuden a la escuela y pueden verse trabajando en las huertas o pidiendo ayuda económica en el centro de los pueblos. El DIF apoya con algunas despensas, cobijas y vestimenta.

Las familias indígenas migrantes en el estado de Chihuahua se enfrentan a condiciones de marginación, pobreza y desigualdad. Existe evidencia en los relatos y análisis descriptivo de que el tamaño del núcleo familiar es determinante de dichas condiciones. Las familias con más miembros presentan una mayor marginación y pobreza, además, permiten el trabajo de niños menores de 14 años de edad y mayores de siete. Además, el ambiente de trabajo en las huertas privadas y en el mercado informal se caracteriza por ser para hombres, dónde las mujeres enfrentan situaciones de hostigamiento e incluso abuso sexual, por lo que casarse es 
una táctica para intentar ser respetada. La sumisión femenina y el silencio sobre las injusticias y el irrespeto hacia la mujer caracterizan los relatos de las propias mujeres, quienes aceptan como parte de su identidad, la victimización de la violencia contra ellas.

Así, la situación de las mujeres indígenas queda sujeta a las desigualdades lingüísticas, educativas, políticas y económicas entre los sexos. Además de diversas representaciones y construcciones mentales sobre las mujeres que terminan por generar desigualdad, abuso y violencia.

\section{Conclusión}

Las NNA indígenas migrantes tienen pocas posibilidades para vivir su infancia con seguridad, dignidad y libertad, pues, inician a una edad temprana labores complejas de trabajo doméstico y vida sexual, responsabilidad en la alimentación de cerdos, chivos y gallinas, así como atención a bebés que disminuyen el tiempo de juego y, por ende, las oportunidades para ser alegres, tener un pensamiento imaginativo-creativo, adquirir condiciones socio-culturales básicas, introducirse a las reglas sociales de la vida y contar con diversas experiencias educativas necesarias para asumir el complejo rol de la ciudadanía femenina. Mientras que los niños aprenden labores relacionadas con el campo y asumen conductas violentas, ilegales o deshonestas sin importar la responsabilidad penal, legal y social de estos actos. El trabajo infantil, aunado con la marginación, pobreza, mala alimentación y falta de higiene ocasionan problemas de tartamudez, perceptivo-foniátricos, comunicativo-lingüísticos, cognitivo-matemáticos y de asociación de palabras-contexto según pruebas realizadas por el grupo de investigación en la Unidad Técnico-Experimental en Fonoaudiología, Semántica-Léxica y Didáctica Musical de Conacyt en 2016. Además, la infancia masculina dura pocos años, pues, los adolescentes son reclutados por las organizaciones criminales en estos municipios para participar en el narcotráfico, el sicariato, la trata de mujeres, la prostitución de mujeres y la pornografía infantil.

Fotografía 2. Niño con arma de fuego

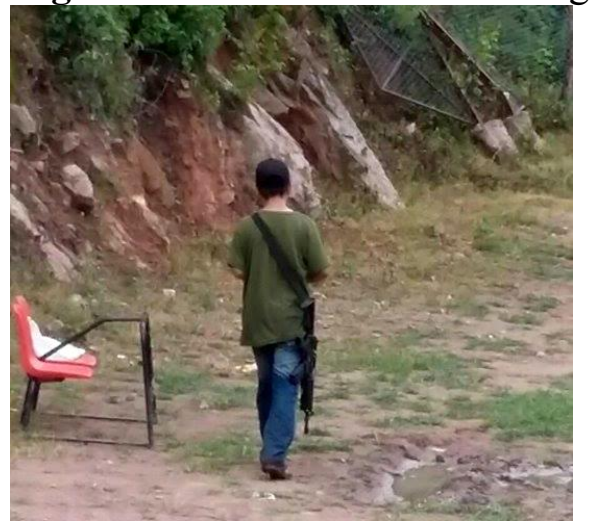

Fuente: Fotografía tomada en uno de los municipios

\section{Referencias}


Aguirre, J. y Jaramillo, L. (2012). Aportes del Método Fenomenológico a la Investigación Educativa. Revista Latinoamericana de Estudios Educativos, vol. 8, núm. 2, Pp. 51 74. Colombia: Universidad de Caldas, Manizales. Recuperado de: https://www.redalyc.org/pdf/1341/134129257004.pdf

Ander-Egg, E. (1995). Técnicas de Investigación Social. Argentina: Lumen.

Arias, F. (1981). Introducción a la Técnica de Investigación en Ciencias de la Administración y del Comportamiento. México; Trillas, S.A.

Coordinación Estatal de la Tarahumara (CET, 2010). Pueblos indígenas de la sierra tarahumara. Recuperado de: http://www.chihuahua.gob.mx/atach2/tarahumara/uploads/Rese\%F1a\%20Ind\%ED gena.pdf.

De la O, M. y Flores, A. (2012). Violencia, jóvenes y vulnerabilidad en la frontera noreste de México. Desacatos, Revista de Antropología Social, núm. 38, Pp. 11-28. México: Centro de Investigaciones y Estudios Superiores en Antropología Social (CIESAS). Recuperado de: http://desacatos.ciesas.edu.mx/index.php/Desacatos/issue/view/15

Garduño, E., Mata, C. y Navarro, A. (2010). Música y visibilidad de los mixtecos en San Quintín, Baja California. Revista Chilena de Antropología Visual, núm. 16, Pp. 133163. Chile: Subdirección de Investigación del Servicio Nacional del Patrimonio Cultural, perteneciente al Ministerio de las Culturas, las Artes y el Patrimonio. Recuperada de: http://www.antropologiavisual.cl/garduno_mata_\&_navarro.htm

Gómez, C. (2018). Los derechos de las niñas, niños y adolescentes (NNA) no acompañados en Caravanas Migrantes en su paso por México. Senado de la República en la LXIV Legislatura/ Serie Mirada Legislativa. Núm. 164, Pp. 1-39. México: Dirección General de Análisis Legislativo/ Instituto Belisario Domínguez. Recuperado de: http://bibliodigitalibd.senado.gob.mx/handle/123456789/4299

Instituto Nacional para la Evaluación de la Educación (INEE, 2015). La Población Indígena en México. México: Estadísticas continuas del formato 911 (inicio del ciclo escolar 2013-2014), SEP-DGPEE y en el Censo de Escuelas, Maestros y Alumnos de Educación Básica y Especial 2013 (CEMABE), INEGI-SEP. Recuperado de: http://publicaciones.inee.edu.mx/buscadorPub/P2/A/310/P2A310.pdf

Ley de la Coordinación Estatal de la Tarahumara (1987). Recuperado de: http://www.redindigena.net/leyes/mex/esta/doc/TARAHUCHIH.htm

Martínez, M. (1999). La Investigación Cualitativa Etnográfica en Educación: Manual Teórico-Práctico. México: Editorial Trillas.

Moctezuma, M. (2018). Menores inmigrantes vulnerados por el gobierno estadounidense. Atrocidades y omisiones de las políticas públicas. Revista Papeles de Población, vol. 24, núm. 98, Pp. 133-156. Recuperado de: https://rppoblacion.uaemex.mx/article/view/11335

Morales, B. (2019). Reflexiones sobre la migración a partir de Los niños perdidos de Valeria Luiselli. Diseminaciones, Revista de Investigación y Crítica en Humanidades y Ciencias Sociales, vol. 2, núm. 3, Pp. 53-70. México: Universidad Autónoma de Queretaro. Recuperado de: http://diseminaciones.uaq.mx/index.php/ojs/article/view/29

ONU (2012). Informe de ONU pone a Tarahumaras como los más desprotegidos de México; desde 2008 alerta abandono. Recuperado de: http://www.sinembargo.mx/20-01$2012 / 125857$ 
Prado, G. (2017). Los derechos humanos de los migrantes. Revista Perfiles de las Ciencias Sociales, núm. 8, Pp. 1-31. México: Universidad Juárez Autónoma de Tabasco. Recuperado de: http://www.revistas.ujat.mx/index.php/perfiles/article/view/3142/2358

Sautú, R. (Comp., 1999). El método biográfico. La reconstrucción de la sociedad a partir del testimonio de los actores. Argentina: Editorial de Belgrano

Velasco, L. y Rentería, D. (2019). Diversity and interculturality: The indigenous school in the context of migration. Revista Estudios Fronterizos, vol. 20, Pp. 1-28. México: Universidad Autónoma de Baja California. Recuperado de: http://www.scielo.org.mx/scielo.php?pid=S018769612019000100101\&script=sci_arttext\&tlng=en 